\title{
The analysis and design of multi-layer microstrip moisture sensor for rice grain
}

\begin{abstract}
The analysis and optimal design of a multi-layered microstrip sensor for measuring moisture content of ricegrain are described. The microstrip sensing structure consists of three layers: substrate, protective layer and semi-infinite grain medium. The effective dielectric constant, characteristic impedance and attenuation or insertion loss of this structure are calculated at various moisture contents with respect to the thickness of the protective layer thickness. The sensor is operated at $9 \mathrm{GHz}$ and is suitable for a broad range of moisture contents (MC) ranging from $10 \%$ to $30 \%$ (wet basis). This study investigated the optimal thickness of the protective layer for suitable sensitivity, the thickness of grain medium which can be consider as semi-infinite thickness, the effect of kernel density and loading method. The experimental results for the attenuation of the signal at various moisture contents agree reasonably with the theoretical prediction however for better accuracy, the compactness of grain medium should be observed. The effect of air inside the medium and compactness of the material under the test has been studied and the errors of $\pm 0.16 \mathrm{~dB}$ have been calculated for the measured reflected attenuation of the sensor. The overall accuracy of the sensor is about $\pm 1.5 \%$ moisture content as compared to oven drying method.
\end{abstract}

Keyword: Multi-layer microstrip sensor; Moisture content; Dielectric constant 